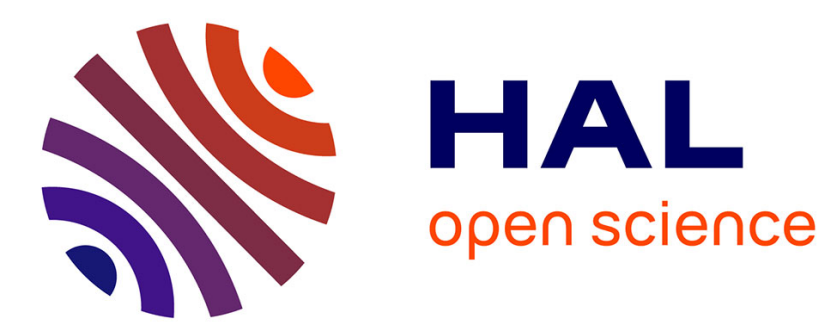

\title{
La spectroscopie des électrons Auger
}

Jean-Paul Deville

\section{To cite this version:}

Jean-Paul Deville. La spectroscopie des électrons Auger. Revue de Physique Appliquée, 1968, 3 (4), pp.351-355. 10.1051/rphysap:0196800304035100 . jpa-00242871

\section{HAL Id: jpa-00242871 https://hal.science/jpa-00242871}

Submitted on 1 Jan 1968

HAL is a multi-disciplinary open access archive for the deposit and dissemination of scientific research documents, whether they are published or not. The documents may come from teaching and research institutions in France or abroad, or from public or private research centers.
L'archive ouverte pluridisciplinaire HAL, est destinée au dépôt et à la diffusion de documents scientifiques de niveau recherche, publiés ou non, émanant des établissements d'enseignement et de recherche français ou étrangers, des laboratoires publics ou privés. 


\title{
LA SPEGTROSGOPIE DES ÉLEGTRONS AUGER
}

\author{
Par Jean-Paul DeVille, \\ Laboratoire de Minéralogie et de Pétrographie, Faculté des Sciences de Strasbourg, I, rue Blessig, 67-Strasbourg.
}

\begin{abstract}
Résumé. - Nous décrivons une nouvelle méthode d'analyse des couches superficielles. Cette méthode est basée sur l'observation de maxima subsidiaires dans les spectres d'énergie des électrons secondaires émis par des cibles bombardées par des électrons. Ces maxima sont dus aux électrons Auger. Après avoir rappelé quelques notions fondamentales sur l'émission secondaire des électrons, nous décrivons un appareil permettant une bonne résolution des pics Auger. Enfin, nous exposons les travaux déjà faits avec cette technique. Ils montrent qu'elle détecte très bien les éléments légers et qu'elle peut déterminer quantitativement les impuretés superficielles.
\end{abstract}

Abstract. - We describe a new method of analysis of surface layers. This method uses the observation of subsidiary maxima in the energy spectra of secondary electrons emitted by electron bombarded targets. Auger electrons are responsible of these maxima. After having summarized some fundamentals of secondary electron emission, we describe an apparatus giving good resolution of Auger peaks. Finally, we report the work already carried out using this technique. They show that it is a sensitive detector for light elements and that it can be a quantitative method of determining surface impurities.

Récemment, une technique d'analyse des couches superficielles a été mise au point. Elle est basée sur l'étude des spectres d'énergie des électrons secondaires émis par une substance lorsqu'elle est frappée par un faisceau d'électrons primaires. Jusque-là, elle a surtout servi d'auxiliaire à la diffraction des électrons lents, mais elle peut être intéressante dans bien d'autres domaines.

I. L'émission secondaire. - Lorsqu'un faisceau d'électrons frappe une cible quelconque, cristalline ou non, la cible émet des électrons secondaires d'énergies diverses. On peut déterminer la distribution en énergie de ces électrons par différentes méthodes. Nous en décrirons deux succinctement, de nombreux articles de synthèse $[1,2]$ existant par ailleurs dans ce domaine.

a) MÉthode du potentiel Retardateur (fig. 1). - Un canon à électrons $A$ envoie sur une cible $B$ des électrons d'énergie $E_{\mathrm{p}}$. Les électrons secondaires émis par la cible sont alors attirés par un collecteur $\mathrm{C}$. Pour faire la discrimination des énergies, on place entre la cible et le collecteur une grille $\mathrm{D}$ portée à un potentiel retardateur $E_{\mathrm{p}}-E^{\prime}$. Seuls les électrons d'énergie supérieure à $E_{\mathrm{p}}-E^{\prime}$ pourront franchir cette barrière de potentiel. En mesurant l'intensité recueillie sur le collecteur en fonction du potentiel de la grille,

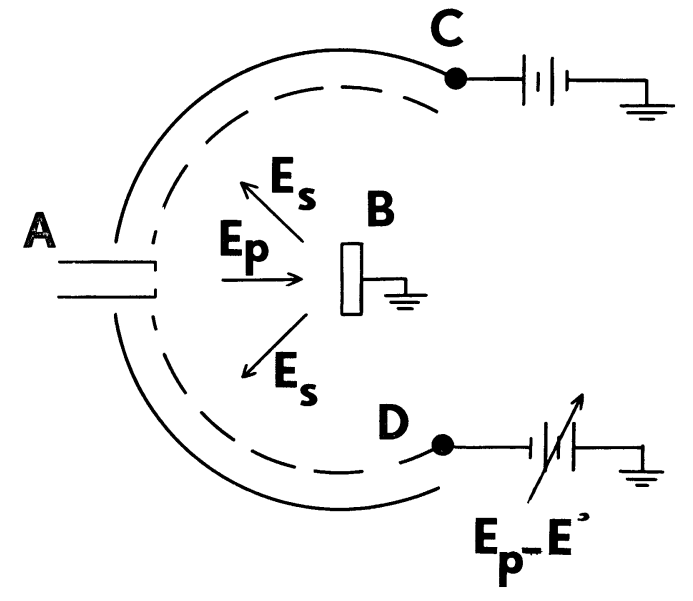

FIG. 1.

on obtient une courbe cumulative de la répartition de l'énergie des électrons secondaires. Sa dérivée donne le nombre d'électrons ayant une énergie donnée $E$.

b) MÉthode DU GHamp ÉLEGTRIQUe tRansVersal (fig. 2). - Les énergies des électrons secondaires émis par la cible sont analysées par un secteur cylindrique de $127^{\circ}$. Ce type d'analyseur focalise sur la fente de 


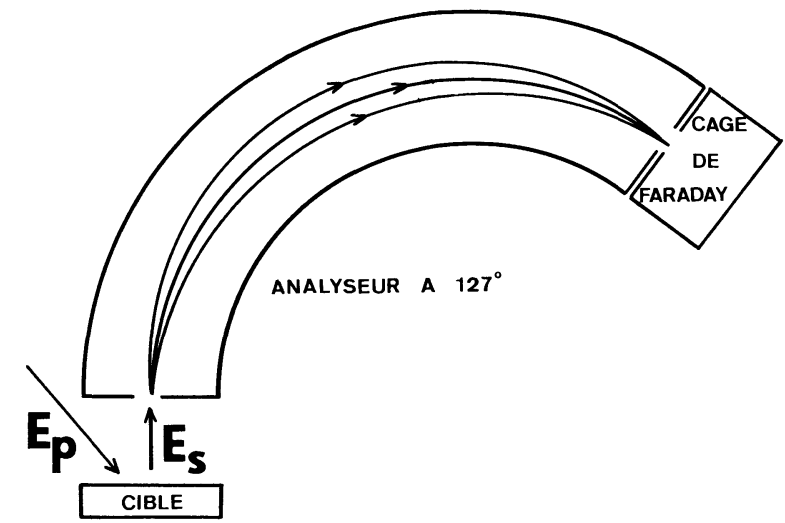

FIG. 2.

sortie les électrons issus de la fente d'entrée ayant même énergie. Cette énergie $E_{\mathrm{s}}$ est liée aux constantes de l'appareil par la relation :

$$
E_{\mathrm{s}}=\frac{1}{\operatorname{Ln}(b / a)} E
$$

où $b$ et $a$ sont les rayons de courbure des deux armatures du condensateur cylindrique et $E$ la différence de potentiel entre ces deux armatures. L'intensité du courant des électrons qui sortent de l'analyseur est mesurée par une cage de Faraday.

Les courbes de distribution des énergies des électrons secondaires obtenues grâce à ces méthodes présentent une allure générale ( $f g .3$ ).

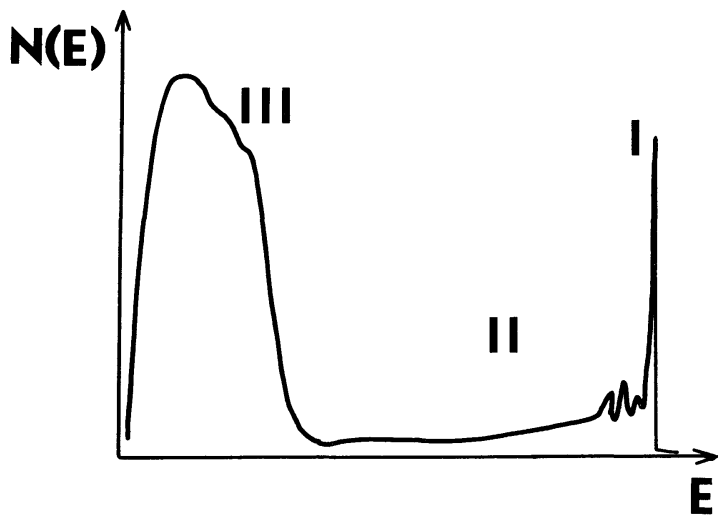

FIG. 3.

Dans cette courbe, on peut distinguer trois régions :

I : Région relative aux électrons primaires qui n'ont pas subi de pertes d'énergie et qui ont donc été diffusés élastiquement. Il y a un maximum aigu pour $E=E_{\mathrm{p}}$ si $E_{\mathrm{p}}$ est l'énergie du faisceau primaire.

II : Région relative aux électrons primaires rediffusés ayant subi des pertes d'énergie.

III : Région relative aux électrons secondaires proprement dits. On peut observer dans cette région un maximum très important, en général situé entre 2 et $3 \mathrm{~V}$.
On observe dans les régions II et III des maxima secondaires dont l'étude est très intéressante car ils sont caractéristiques de la cible.

Les maxima de la région II correspondent à des pertes discrètes d'énergie, indépendantes de l'énergie du faisceau primaire mais dépendantes de la nature de la cible. Ces pertes d'énergie ont été interprétées par Harrower [3], [4] comme résultant de transitions interbandes. En plus de ces transitions, il faut aussi tenir compte des pertes résultant de l'excitation d'oscillations de plasma.

Les maxima de la région III correspondent à des énergies données, caractéristiques de la cible, indépendantes de l'énergie du faisceau primaire. On peut voir sur la figure 3 que ces maxima n'apparaissent en général que sous la forme de points d'inflexion : en effet, ils sont de faible intensité et sont masqués par le maximum important des électrons secondaires proprement dits. Lander [5] les a interprétés en 1953 comme résultant de transitions Auger [6].

Il est évident que cette distinction en trois régions est relativement arbitraire. On trouve dans la région I des maxima dus à des pertes très faibles liées aux excitations « phonons ». En plus, les différentes pertes peuvent coexister dans les trois régions.

En utilisant un modèle de bandes d'énergie, Lander et Harrower ont donné une explication simple des maxima dus aux transitions Auger (fig. 4).

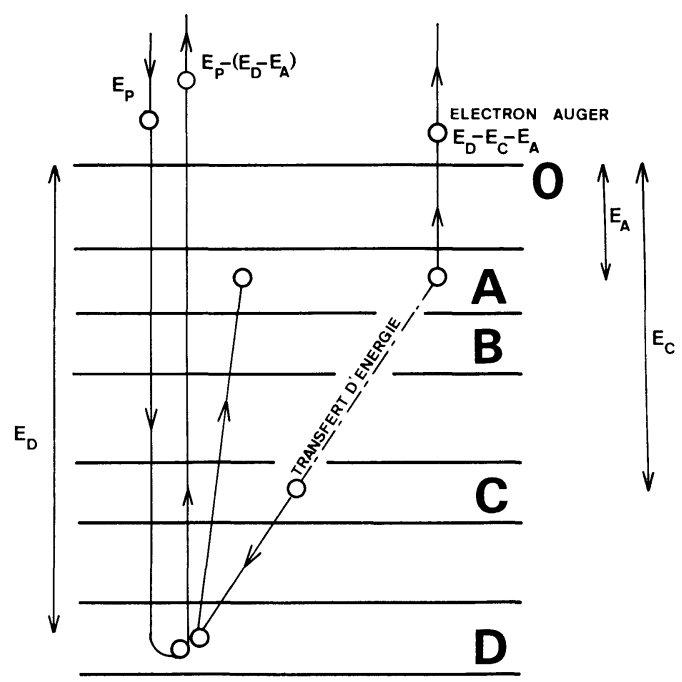

FIG. 4.

$\mathrm{A}, \mathrm{B}, \mathrm{C}$ et $\mathrm{D}$ sont, pour un atome donné, des bandes d'énergie de plus en plus profondes et $\mathrm{O}$ est le potentiel $\mathrm{du}$ vide. Considérons un électron du faisceau primaire ayant une énergie $E_{\mathrm{p}}$. Il pénètre dans le réseau et communique à un électron de la bande $\mathrm{D}$ une énergie $E_{\mathrm{D}}-E_{\mathrm{A}}$. L'électron $\mathrm{D}$ va donc aller au niveau $\mathrm{A}$, tandis que l'électron primaire va ressortir après avoir perdu l'énergie $E_{\mathrm{D}}-E_{\mathrm{A}}$.

Ce schéma explique les pertes discrètes au voisinage 
du pic élastique, pertes égales à $E_{\mathrm{D}}-E_{\mathrm{A}}, E_{\mathrm{C}}-E_{\mathrm{A}} \ldots$ que l'on peut retrouver sur la courbe de la figure 3 .

L'électron qui a quitté le niveau $\mathrm{D}$ y a laissé une case libre; cette case pourra être occupée par un électron de la bande $\mathrm{G}$. Ce transport d'électrons libère une énergie $E_{\mathrm{D}}-E_{\mathrm{c}}$. Au lieu de se libérer sous forme de rayonnement $\mathrm{X}$, cette énergie peut être transmise à un autre électron, par exemple un électron du niveau A qui va sortir de l'atome avec l'énergie $\left(E_{\mathrm{D}}-E_{\mathrm{C}}\right)-E_{\mathrm{A}}$ sans que l'on connaisse le mécanisme exact de ce transfert d'énergie. Si l'énergie était transmise à un électron du niveau $\mathrm{C}$, celui-ci sortirait avec l'énergie $\left(E_{\mathrm{D}}-E_{\mathrm{C}}\right)-E_{\mathrm{C}}=E_{\mathrm{D}}-2 E_{\mathrm{C}}$. Ces processus donnent, selon un mécanisme Auger, des électrons secondaires dont l'énergie est caractéristique de l'atome constituant la cible.

Lander et Harrower ont vérifié l'exactitude de ce mécanisme à partir des données énergétiques des niveaux atomiques utilisées pour les spectres d'absorption des rayons $\mathrm{X}$ [7], [8]. Ils ont trouvé, à quelques volts près, les valeurs $E_{\mathrm{D}}-E_{\mathrm{C}}-E_{\mathrm{A}}, E_{\mathrm{D}}-2 E_{\mathrm{C}} \ldots$ pour les transitions de type Auger. De plus, ils ont constaté que, contrairement à l'émission de rayonnement X, l'émission d'électrons Auger n'obéissait pas à des règles de sélection très strictes. Cependant, certaines transitions sont beaucoup plus probables que d'autres [9] et certaines émissions sont en général expérimentalement invisibles. De toute façon, les spectres d'énergie sont caractéristiques de la nature de l'atome ayant émis les électrons Auger et pourront servir à son identification. Nous signalons aussi un ouvrage traitant en détail de l'effet Auger [10].

Il convient de remarquer que ce mécanisme de transitions individuelles est satisfaisant pour l'interprétation des maxima subsidiaires situés dans la région III de la courbe de distribution des énergies des électrons secondaires qui sont les maxima étudiés dans la spectroscopie des électrons Auger. Cependant, ce mécanisme n'est pas suffisant pour expliquer les maxima de la région II et il est impossible de négliger l'interprétation qui en a été donnée par Pines et Bohm [11]. Il faut avoir recours à la notion d'oscillations collectives du plasma. Si la répartition des charges qui assure la neutralité électrique du plasma est perturbée par une cause quelconque (par exemple par la traversée d'un électron provenant du faisceau primaire), les électrons du plasma vont se déplacer pour rétablir la neutralité électrique; il en résulte un mouvement oscillatoire des électrons de fréquence $\nu_{p}$. On aura les relations :

$$
\omega_{\mathrm{p}}=2 \pi \nu_{\mathrm{p}}=\left(\frac{4 \pi n e^{2}}{m}\right)^{1 / 2}
$$

où $n$ est le nombre d'électrons libres du système considéré. Par l'intermédiaire de ce nombre $n$, la fréquence $\nu_{p}$ est caractéristique de la cible. Les pertes d'énergie appelées " caractéristiques » seront alors égales à $\hbar \omega_{p}, 2 \hbar \omega_{p} \ldots$

De plus, Ritchie [12] a mis en évidence l'existence d'oscillations de surface d'énergies légèrement différentes qui dépendent beaucoup de l'état de propreté de la surface.

Pour une étude plus approfondie des « pertes caractéristiques ", nous renvoyons au récent ouvrage de Gauthé [13].

\section{Application de la spectroscopie des électrons} Auger aux problèmes d'études des surfaces. - Déjà en 1953, Lander prévoyait que l'analyse des maxima dus aux électrons Auger pouvait être une technique puissante pour l'étude des surfaces, mais jusqu'à présent les nécessités techniques (ultravide, appareillage électronique important) avaient empêché de faire ces études.

Récemment, Tharp et Scheibner [14], [15], ainsi que Harris [16], [17] ont montré que le phénomène d'émission d'électrons Auger était extrêmement sensible à la composition des toutes premières assises atomiques, et en particulier à celle des couches de contamination. Gela peut se comprendre assez facilement : comme tous les électrons de faible énergie ont une faible pénétration, les électrons Auger, qui ont une énergie ne dépassant $500 \mathrm{eV}$ que dans des cas très rares, ne pourront sortir du solide que s'ils proviennent d'atomes peu éloignés de la surface. On a donc ainsi un moyen d'étude des couches superficielles.

Harris a utilisé pour cette spectroscopie un système d'analyse des énergies des électrons secondaires basé sur la méthode du champ électrique transversal, tandis que Tharp et Scheibner utilisaient l'optique électronique d'un diffractographe à électrons lents. En effet, le montage des électrodes dans cet appareil est très analogue à celui utilisé dans la méthode du champ retardateur : le collecteur sera l'écran fluorescent et la grille de filtrage la grille suppresseuse. On obtient ainsi une courbe $I=f(E)$ où $I$ est le courant recueilli par l'écran collecteur et $E$ le potentiel de la grille de filtrage. Cette courbe est la courbe cumulative des électrons secondaires en fonction de l'énergie.

Pour obtenir la courbe dérivée $\frac{\mathrm{d} I}{\mathrm{~d} E}=N(E)$, c'està-dire le nombre d'électrons ayant l'énergie $E$, on superpose à la tension continue variable de la grille de filtrage un petit signal alternatif. On voit facilement

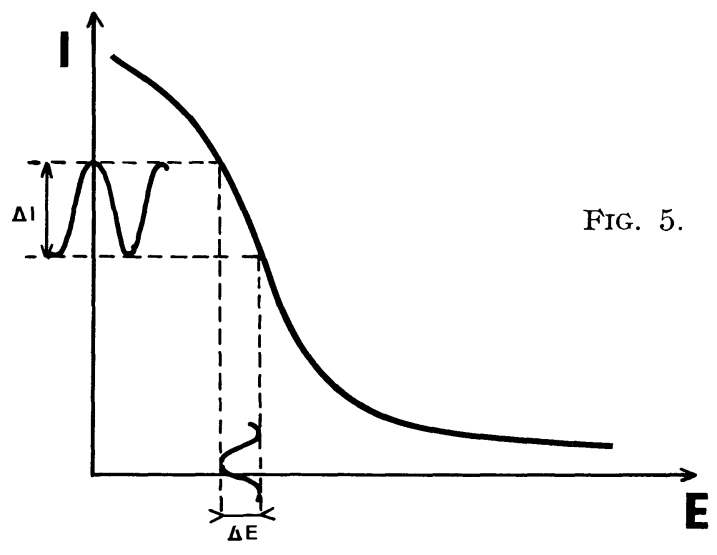


sur la figure 5 que le courant alternatif qui sera collecté est proportionnel à la pente de la courbe $I=f(E)$. Par ce moyen, on obtient la courbe décrite sur la figure 3 . Comme il est difficile sur cette courbe de bien situer la position des transitions Auger qui n'apparaissent que comme des points d'inflexion, il sera intéressant de procéder à une nouvelle dérivation. Cela est possible en mesurant la deuxième harmonique du signal alternatif reçu par le collecteur. En effet, développons l'intensité $I$ en série de Taylor au voisinage du potentiel retardateur $E$ :

$$
\begin{aligned}
I(E+\Delta E) & =I(E)+\Delta E \frac{\mathrm{d} I(E)}{\mathrm{d} E} \\
+ & \frac{1}{2}(\Delta E)^{2} \frac{\mathrm{d}^{2} I(E)}{\mathrm{d} E^{2}}+\frac{1}{6}(\Delta E)^{3} \frac{\mathrm{d}^{3} I(E)}{\mathrm{d} E^{3}}+\ldots
\end{aligned}
$$

Posons $\Delta E=k \sin \omega t \quad$ (signal alternatif de faible amplitude superposé à $E$ que nous appellerons le signal de modulation). Il vient :

$$
\begin{aligned}
I(E+\Delta E) & =I(E)+k N(E) \sin \omega t \\
& +\frac{k^{2}}{4} \frac{\mathrm{d} N(E)}{\mathrm{d} E}(1-\cos 2 \omega t) \\
& +\frac{k^{3}}{24} \frac{\mathrm{d}^{2} N(E)}{\mathrm{d} E^{2}}(3 \sin \omega t-\sin 3 \omega t)+\ldots
\end{aligned}
$$

avec $N(E)=\mathrm{d} I(E) / \mathrm{d} E$.

On voit que, si la tension de modulation $k$ sin $\omega t$ est suffisamment faible pour que l'on puisse se limiter à un développement d'ordre 3 , le coefficient de la deuxième harmonique est proportionnel à $\mathrm{d} N(E) / \mathrm{d} E$.

Ces différentes opérations peuvent être réalisées par le montage suivant que nous allons décrire ( $f g .6$ ).

Un canon à électrons $\mathrm{A}$ envoie un faisceau d'électrons sur la cible B. Les électrons secondaires sont attirés sur l'écran collecteur $\mathrm{C}$ par une tension continue d'environ $300 \mathrm{~V}$. La grille de filtrage D est portée au potentiel retardateur grâce à une alimentation en courant continu dont la tension de sortie est commandée par un moteur. On superpose à travers un transformateur d'isolement la tension alternative de pertur-

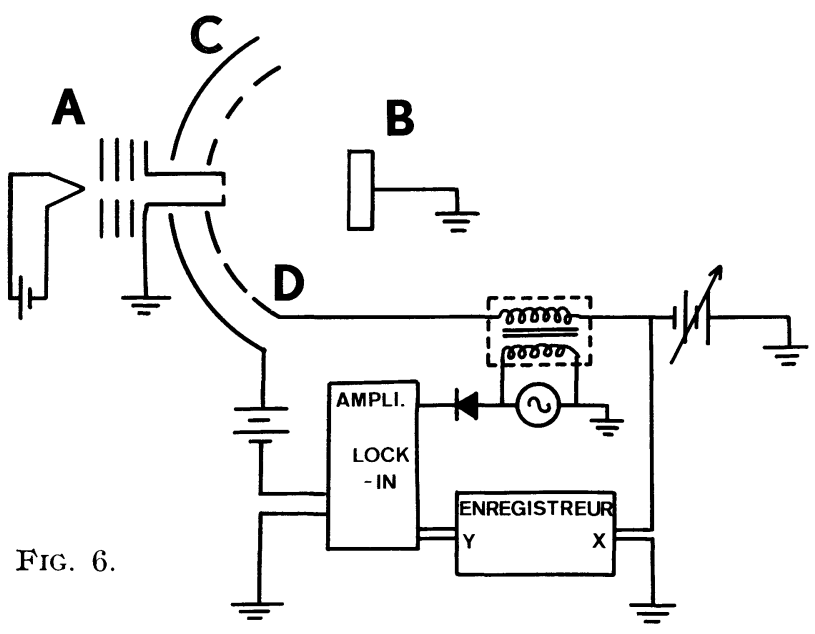

bation (environ $435 \mathrm{~Hz}$ ) à la tension retardatrice continue pour obtenir la première dérivation. D'autre part, le signal alternatif est envoyé à travers une diode sur le canal de référence d'un amplificateur synchrone (type Lock-in). Cet amplificateur permet de sélectionner, dans le courant qu'il reçoit par ailleurs du collecteur, uniquement la partie qui a la fréquence affichée au canal de référence et qui est en phase avec le courant appliqué à ce canal de référence. Si l'on affiche au canal de référence une fréquence double de celle appliquée à la grille de filtrage, on mesurera bien avec l'amplificateur Lock-in l'harmonique $2 \mathrm{du}$ courant reçu sur le collecteur. Finalement, le signal mesuré par l'amplificateur Lock-in est envoyé en Y d'un enregistreur XY, tandis que la borne $\mathrm{X}$ est reliée à la tension retardatrice variable.

Il faut noter que, pour avoir un bon rendement d'électrons Auger, il est nécessaire d'exciter les niveaux profonds des atomes. Pour cela, il faudra avoir une tension du faisceau primaire allant de 1500 à $2000 \mathrm{~V}$. De plus, comme les signaux reçus sont faibles, il faudra éviter tous les couplages entre la grille et l'écran et donc faire un blindage extrêmement soigné de toutes les connexions. Toutes les mesures devront être faites dans l'ultravide de façon à éviter les perturbations dues à la contamination.

III. Résultats obtenus. - Mis à part les quelques travaux des années 1953 à 1956 dus à Lander [5], Harrower [3], [4], Zinke [18] et autres, les études de spectroscopie des électrons Auger sont extrêmement récentes (1967). Aussi y a-t-il encore peu de travaux publiés, bien que cette technique soit en grand développement aux États-Unis. Cependant, elle a déjà été employée avec succès dans deux domaines : d'une part, l'analyse des surfaces des métaux polycristallins et, d'autre part, comme auxiliaire de la diffraction des électrons lents.

Tharp et Scheibner [14] ont étudié la contamination du tungstène par le carbone. On sait que le carbone, impureté majeure du tungstène, diffuse depuis la masse du cristal jusqu'à la surface lorsque le cristal est chauffé à haute température de sorte que le chauffage, processus de nettoyage classique des surfaces, ne favorise pas, bien au contraire, la décontamination. Une fois le carbone arrivé à la surface, il faut le brûler dans l'oxygène. Cette méthode de décontamination avait déjà été étudiée grâce au couplage de la spectrométrie de masse et de la diffraction des électrons lents [19]. La technique de spectroscopie des électrons Auger a confirmé ces résultats. Elle a aussi montré qu'après l'oxydation du carbone superficiel il restait encore beaucoup de carbone dans les couches plus internes. Lors du chauffage suivant, le carbone reprend sa migration vers la surface. Ce phénomène n'est pas particulier au tungstène et il a été mis en évidence par Vance [20] dans le cas du molybdène. Scheibner et Tharp [15] ont aussi étudié les spectres donnés par le graphite fraîchement clivé et le cuivre. Mais ils n'ont 
pas encore étudié pour ces corps les influences des couches d'adsorption.

Harris [16-17] a étudié les spectres de plusieurs métaux et alliages polycristallins ( $\mathrm{Be}, \mathrm{Fe}, \mathrm{Ni}$, aciers...) et les couches de contamination dues au soufre, au carbone, à l'azote et à l'oxygène. Il a montré que la spectroscopie des électrons Auger était particulièrement adaptée à la détection des éléments légers et qu'elle complétait ainsi parfaitement la fluorescence X. Il a pu détecter dans les aciers et le nickel la présence d'impuretés à très faible teneur $(40 \mathrm{ppm}$ de carbone, $10 \mathrm{ppm}$ de soufre) et montrer lors du chauffage la migration du soufre vers la surface du nickel et de l'antimoine et du chrome à la surface de certains aciers spéciaux. Si l'on peut admettre que la surface joue un rôle analogue à celui des joints de grains [21], ce phénomène de migration pourrait expliquer la fragilisation de certains métaux et la ségrégation.

Weber et Peria [22] ont étudié le dépôt des métaux alcalins sur le silicium et le germanium. Ils ont montré que les pics Auger des métaux alcalins étaient indépendants de la face du substrat sur laquelle avait lieu le dépôt. La structure des couches n'a donc aucun effet sur les transitions Auger. D'autre part, ils ont prouvé que l'analyse, lorsqu'elle était faite avec soin, était quantitative. En faisant des dépôts contrôlés de césium sur le silicium, ils ont observé que la hauteur du pic Auger dû au césium était proportionnelle au taux de recouvrement de la surface par le métal alcalin. Ils ont $\mathrm{pu}$ mesurer 0,1 couche monoatomique, résultat qu'ils auraient amélioré depuis.
Palmberg et Rhodin [23] ont étudié la profondeur à partir de laquelle les électrons Auger peuvent sortir sans subir de nouvelles pertes d'énergie. Ils ont trouvé dans le cas de l'or et de l'argent une profondeur allant de $4 \AA$ à $8 \AA$ pour des électrons de 72 à $362 \mathrm{eV}$. Cela correspond tout à fait aux valeurs trouvées par ailleurs pour la profondeur de pénétration des électrons lents [24]. Ils ont aussi étudié la propreté de la surface de nombreux métaux ( $\mathrm{Ag}, \mathrm{Au}, \mathrm{Pd}, \mathrm{Cu}, \mathrm{Ni}$ ) et ils suggèrent l'étude de métaux voisins dans la classification périodique. En effet, ces métaux doivent avoir des spectres Auger très semblables car leurs niveaux énergétiques sont voisins. La comparaison de leurs spectres doit permettre de distinguer ce qui provient des impuretés inconnues de ce qui est propre au cristal luimême. Finalement, ils ont étudié l'épitaxie de l'or sur l'argent et de l'argent sur l'or et ont mis en évidence des différences de mode de germination. Dans le premier cas, la croissance a lieu couche par couche, alors que dans le deuxième cas il y a formation d'îlots.

Conclusion. - On peut voir grâce à ces exemples que la spectroscopie des électrons Auger peut être une méthode très puissante pour l'étude de nombreux problèmes relatifs à la surface. Il faut noter que cette technique est délicate à mettre en œuvre et qu'on ne peut l'utiliser en toute confiance pour déterminer une impureté spécifique que lorsque le spectre de cette impureté est connu. En revanche, la spectroscopie des électrons Auger peut s'appliquer à des corps polycristallins ou même amorphes et recevoir ainsi de nombreuses applications.

Note de la Rédaction. - La spectroscopie des électrons Auger peut également être effectuée grâce à l'excitation par des rayons X; le spectre Auger est alors analogue au spectre X de fluorescence. (Voir par exemple B. L. Henke, Norelco Rep., 1967, 14, 75, ou « Atomic, molecular and solid state structure studied by means of electron spectroscopy » par Siegbahn et al., Nova Acta Reg. Soc. Scient., Upsal, 1967, 20.)

\section{BIBLIOGRAPHIE}

[1] McKay (K. G.), Advances in Electronics, 1948, 1, 65.

[2] Haymann (P.), Journal des Recherches du C.N.R.S., décembre 1962, 61, 357.

[3] Harrower (G. A.), Phys. Rev., 1956, 102, 340

[4] Harrower (G. A.), Phys. Rev., 1956, 104, 52.

[5] Lander (J. J.), Phys. Rev., 1953, 91, 1382.

[6] Auger (P.), J. Physique Rad., 1925, 6, 205.

[7] HILL (R. D.), ChURCh (E. L.) et MIHEIICH (J. W.), Rev. Sci. Instr., 1952, 23, 523.

[8] SAGEL (K.), Tabellen zur Röntgen-Emissions und Absorptions-Analyse, Springer-Verlag, Berlin, 1959.

[9] PINCHERLE (L.), Nuovo Cimento, 1935, 12, 81.

[10] Burhop (E. H. S.), The Auger Effect and other Radiationless Transitions, Univ. Press, Cambridge, 1952.

[11] BoHm (D.) et Pines (D.), Phys. Rev., 1951, 82, 625 ; 1952, 85, 338; 1953, 92, 609 ; PINEs (D.), Phys. Rev., 1953, 92, 626.

[12] Ritchie (R. H.), Phys. Rev., 1957, 106, 874.

[13] GaUTHÉ (B.), Les pertes d'énergie caractéristiques des électrons dans les solides, Gauthier-Villars, Paris, 1968.
[14] Tharp (I. N.) et Scheibner (E. J.), J. Appl. Phys., 1967, 38, 3320 .

[15] Scheibner (E. J.) et Tharp (L. N.), Surf. Sci., 1967, 8, 247.

[16] Harris (L. A.), G. E. Res. Develop., 1967, nos $67 \mathrm{C} 199$ et $67 \mathrm{C} 201$.

[17] Harris (L. A.), J. Appl. Phys., 1968, 39, 1419, 1428.

[18] ZINke (O. H.), Phys. Rev., 1957, 106, 1163.

[19] Germer (L. H.), Stern (R. M.) et MACrae (A. U.), Metal Surfaces, Robertson (W. D.) et Gjostein (N. A.) édit. (American Scientific Society for Metals, Ohio, 1963, p. 287).

[20] Vance (D. W.), Phys. Rev., 1967, 164, 372.

[21] Handler (P.), Ann. N. Y. Acad. Sci., 1963, 101, 857.

[22] Weber (R. T.) et Peria (W. T.), J. Appl. Phys., 1967, 38, 4355 .

[23] Palmberg (P. W.) et Rhodin (T. N.), J. Appl. Phys., 1968, 39, 2425.

[24] Deville (J.-P.), EberharT (J. P.) et GoldSzTAUb (S.), C. R. Acad. Sci., 1967, 264, 124. 